\title{
Limbal Fibroblasts Maintain Normal Phenotype in 3D RAFT Tissue Equivalents Suggesting Potential for Safe Clinical Use in Treatment of Ocular Surface Failure
}

\author{
Isobel Massie, PhD, Sarah B. Dale, PhD, and Julie T. Daniels, PhD, FSB
}

Limbal epithelial stem cell deficiency can cause blindness, but transplantation of these cells on a carrier such as human amniotic membrane can restore vision. Unfortunately, clinical graft manufacture using amnion can be inconsistent. Therefore, we have developed an alternative substrate, Real Architecture for 3D Tissue (RAFT), which supports human limbal epithelial cells (hLE) expansion. Epithelial organization is improved when human limbal fibroblasts (hLF) are incorporated into RAFT tissue equivalent (TE). However, hLF have the potential to transdifferentiate into a pro-scarring cell type, which would be incompatible with therapeutic transplantation. The aim of this work was to assess the scarring phenotype of hLF in RAFT TEs in hLE + and hLE - RAFT TEs and in nonairlifted and airlifted RAFT TEs. Diseased fibroblasts (dFib) isolated from the fibrotic conjunctivae of ocular mucous membrane pemphigoid (Oc-MMP) patients were used as a pro-scarring positive control against which hLF were compared using surrogate scarring parameters: matrix metalloproteinase (MMP) activity, de novo collagen synthesis, $\alpha$-smooth muscle actin ( $\alpha$-SMA) expression, and transforming growth factor- $\beta$ (TGF- $\beta$ ) secretion. Normal hLF and dFib maintained different phenotypes in RAFT TE. MMP-2 and -9 activity, de novo collagen synthesis, and $\alpha$-SMA expression were all increased in dFib cf. normal hLF RAFT TEs, although TGF$\beta 1$ secretion did not differ between normal hLF and dFib RAFT TEs. Normal hLF do not progress toward a scarring-like phenotype during culture in RAFT TEs and, therefore, may be safe to include in therapeutic RAFT TE, where they can support hLE, although in vivo work is required to confirm this. dFib RAFT TEs (used in this study as a positive control) may be useful toward the development of an ex vivo disease model of Oc-MMP.

\section{Introduction}

$\mathbf{T}$ HE CORNEA IS LOCATED on the front of the eyeball and provides a transparent window to the world. It is a complex, avascular, multilayered tissue consisting of an outermost epithelial cell layer, which protects the underlying stroma, located anterior to the endothelium. ${ }^{1}$ Maintenance of the epithelium is crucial to corneal function and sloughed epithelial cells are replaced by the progeny of limbal epithelial stem cells (LESC). LESC reside within a specialized niche environment, the limbus, located at the vascularized border between the central cornea and conjunctiva. ${ }^{2,3}$ Damage to the limbus and loss of LESC can lead to ocular surface failure and blindness: the cornea becomes vascularized and inflamed and conjunctival epithelial cells migrate to cover the cornea. ${ }^{4}$

One treatment for LESC deficiency is transplantation of cultured human limbal epithelial cells (hLE) on a carrier such as human amniotic membrane (HAM). However, clinical graft manufacture can be inconsistent. ${ }^{5-7}$ Additionally, HAM is intrinsically biologically variable, ${ }^{8}$ supply can be unreliable, and the tissue must be screened before use which has led us, and others, to investigate alternative means of transplanting hLE. Our approach is to utilize a tissue equivalent (TE), Real Architecture for 3D Tissue (RAFT) that consists primarily of Type 1 collagen. ${ }^{9}$

RAFT TEs are produced by gently dehydrating collagen hydrogels and we have previously demonstrated that RAFT TE is excellent for hLE expansion. RAFT TEs may also be cultured at the air/liquid interface (airlifted) to promote epithelial stratification, suggesting that RAFT TEs may be clinically effective toward the treatment of LESC deficiency. ${ }^{9}$ The organization of epithelium cultured on RAFT TE and deposition of basement membrane proteins is further enhanced when pre-expanded human limbal fibroblasts (hLF) are incorporated into RAFT TEs. ${ }^{9}$ In native limbus,

Department of Ocular Biology and Therapeutics, UCL Institute of Ophthalmology, London, United Kingdom.

Abstract Presented at ARVO Annual Meeting, Orlando, FL, 2014. Massie, I., Dale, S.B., Dart, J.K., and Daniels, J.T. Both Healthy and Diseased Fibroblasts Maintain Phenotype in 3D Tissue-Engineered RAFT Constructs Suggesting Potential for Safe Clinical Use and Ex Vivo Disease Modeling, Respectively. Abstract No. 5163. 
hLE are supported by cells located proximally in the underlying stroma and so the supportive role that hLF play in RAFT TEs is perhaps unsurprising as this culture format mimics the in vivo arrangement. ${ }^{1}$

In vivo, stromal keratocytes (specialized corneal fibroblasts) are normally quiescent. ${ }^{10}$ However, these keratocytes can become activated following epithelial injury and associated cytokine release. These activated cells play a role in wound healing and remodel the damaged stroma, restoring transparency. However, these also have the potential to transdifferentiate further to a pro-scarring phenotype. hLF proliferate in vitro, suggesting some degree of activation, which highlights the possibility of further transdifferentiation into a pro-scarring phenotype.

The overall aim of the RAFT therapy is to treat LESC deficiency and although we know that hLF support hLE in RAFT TEs, the potential risk of hLF transdifferentiation meant that further characterization of hLF was needed. The aim of the current work was to investigate whether or not the preexpanded hLF utilized in RAFT TEs exhibit any pro-scarring characteristics, to determine whether or not they may be safe for clinical use. To do so, a pro-scarring positive control cell type was required against which normal hLF could be compared. The ideal positive control would be pro-scarring limbal fibroblasts, but such cells are unavailable as to biopsy them would cause further damage to the patient. Instead, we chose to use a conjunctival fibroblast population isolated from ocular mucous membrane pemphigoid (Oc-MMP) patients. Oc-MMP is a devastating autoimmune disease that can result in blindness due to episodes of inflammation followed by aggressive scarring of the conjunctiva. ${ }^{11}$ Oc-MMP conjunctival fibroblasts are known to maintain some scarring characteristics in vitro ${ }^{12}$ and are found in very close proximity to normal hLF and so we chose to use these as our positive control cell type (for clarity, the different cell types used in this study are summarized in Table 1). The scarring characteristics of normal hLF in RAFT TEs were assessed in the presence and absence of hLE, before and after airlifting.

\section{Materials and Methods}

\section{Chemicals}

All reagents were obtained from Life Technologies, unless stated otherwise.

Table 1. Cell Types Described in This Article

\begin{tabular}{ccc}
\hline Abbreviation & Meaning & \multicolumn{1}{c}{ Definition } \\
\hline hLE & $\begin{array}{c}\text { Human limbal } \\
\text { epithelial cell }\end{array}$ & $\begin{array}{c}\text { Limbal epithelial cell } \\
\text { isolated from the } \\
\text { limbus of normal } \\
\text { donors }\end{array}$ \\
$\begin{array}{c}\text { Normal } \\
\text { hLF }\end{array}$ & $\begin{array}{c}\text { Normal human } \\
\text { limbal } \\
\text { fibroblast } \\
\text { Diseased } \\
\text { fibroblast }\end{array}$ & $\begin{array}{c}\text { fromal cell isolated } \\
\text { for normal donors } \\
\text { oell with pro-scarring } \\
\text { characteristics isolated } \\
\text { from the conjunctiva } \\
\text { of ocular mucous } \\
\text { membrane pemphigoid } \\
\text { patients }\end{array}$ \\
& & \\
& &
\end{tabular}

\section{Human cell isolation and culture}

Normal donor tissue. Cadaveric donor corneal rims with appropriate research consent were obtained from the Moorfields Lions Eye Bank (United Kingdom). Ethical permission for this study was obtained from the Research Ethics Committee (United Kingdom) (Ref. No. 10/H0106/57-11ETR10). Corneas were stored at $4^{\circ} \mathrm{C}$ in Optisol (Chiron Ophthalmics, Inc.) after enucleation and before hLE and normal hLF isolation.

Diseased donor tissue (for positive control). Diseased conjunctival biopsies were taken from patients with biopsy positive (direct immunofluorescence) Oc-MMP, presenting with active conjunctival scarring. Ethical permission was obtained from the Research Ethics Committee (United Kingdom) (Ref No. 10/H0311/40). Biopsies were stored at $4^{\circ} \mathrm{C}$ in L-15 medium (Leibovitz's) until diseased fibroblast (dFib) isolation.

Isolation and culture of normal hLE. hLE were isolated and cultured as described previously. ${ }^{9}$ Briefly, dispase digestion and mechanical scraping was used to isolate hLE from donor corneal rims. hLE were cultured in corneal epithelial culture medium (CECM) on growth-arrested 3T3$\mathrm{J} 2$ cells and passaged using $0.5 \%$ trypsin-EDTA before seeding onto RAFT TE at passage 1.

Isolation and culture of normal hLF. After hLE isolation, the remaining scleral-limbal rim quarters were trimmed of excess sclera and cut into three pieces. These were placed in explant culture with hLF culture medium (DMEM basal medium, $10 \%$ fetal bovine serum, $1 \%$ AA) in a humidified $5 \% \mathrm{CO}_{2}$ in air incubator at $37^{\circ} \mathrm{C}$. Explant cultures were maintained until hLF outgrowth was observed before being passaged using $0.05 \%$ trypsin-EDTA. hLF were not used beyond passage 6 .

Isolation and culture of diseased (positive control) human conjunctival fibroblasts. Biopsies were placed in $1.2 \mathrm{IU} / \mathrm{mL}$ dispase II for $1 \mathrm{~min}$ and epithelium scraped away. Small cuts were made in the biopsies to encourage dFib outgrowth and biopsies were placed in explant culture with hLF culture medium in a humidified $5 \% \mathrm{CO}_{2}$ in air incubator at $37^{\circ} \mathrm{C}$. hLF medium was changed once per week until outgrowth was observed. dFib were not used beyond passage 6 .

\section{Preparation of RAFT TE}

Normal hLF or dFib were suspended at $5.8 \times 10^{4} / \mathrm{mL}$ final collagen mix in hLF medium. Collagen mix was prepared by mixing 1 part $10 \times$ MEM, 8 parts $2 \mathrm{mg} / \mathrm{mL}$ sterile rat-tail Type I collagen (TAP BioSystems), and 0.59 parts neutralizing solution (TAP BioSystems) to achieve a $\mathrm{pH} \sim 7.2$. Fibroblast cell suspension (0.41 parts) was added, mixed, and left on ice for $30 \mathrm{~min}$ to allow any small bubbles to disperse. This mixture $(2.4 \mathrm{~mL})$ was pipetted into each well of a 24-well plate (Greiner Bio-One) and placed on a heater (TAP BioSystems) at $37^{\circ} \mathrm{C}$ for $30 \mathrm{~min}$ to undergo fibrillogenesis. Water was wicked away from the collagen hydrogels through application of hydrophilic porous absorbers (TAP BioSystems). ${ }^{13}$ After $30 \mathrm{~min}$, the absorbers were removed and $2 \mathrm{~mL}$ of hLF medium was added. After $2 \mathrm{~h}$, if hLE were 
required, $5.8 \times 10^{5} \mathrm{hLE}$ suspended in $2 \mathrm{~mL}$ of CECM were seeded onto each RAFT TE (when not required, hLF medium was replaced with $2 \mathrm{~mL}$ of CECM). RAFT TEs were maintained in a humidified $5 \% \mathrm{CO}_{2}$ in air incubator at $37^{\circ} \mathrm{C}$, with medium changes three times per week, for 2 weeks.

\section{Airlifting to induce stratification of $h L E$}

To determine whether the scarring profiles of normal hLF was affected by the presence of a monolayer of epithelial cells or multilayered epithelium, some RAFT TEs were airlifted.

RAFT TEs were carefully transferred to a cell culture insert (Millipore) in six-well plates using a spatula and forceps. CECM $(800 \mu \mathrm{L})$ was added underneath each insert to maintain hLE at the air-liquid interface. Airlifted RAFT TEs were maintained using CECM in a humidified $5 \% \mathrm{CO}_{2}$ in air incubator at $37^{\circ} \mathrm{C}$ for 1 week.

\section{Preparation of conditioned medium}

RAFT TEs containing either normal hLF or dFib, with and without hLE, and with and without airlifting were washed six times with phosphate buffered saline (PBS) and then serum-starved for $18 \mathrm{~h}$ in $2 \mathrm{~mL}$ of serum-free medium (0.1\% BSA [Sigma-Aldrich], $1 \%$ AA). Serum starving was necessary as the serum in CECM used to culture all RAFT TEs contains growth factors and enzymes that may impact on quantitative analysis. Acellular (no fibroblasts or hLE) RAFT TEs were included as negative controls. Conditioned medium was collected, aliquoted, and stored at $-80^{\circ} \mathrm{C}$ until analysis.

\section{Matrix metalloproteinase activity}

Matrix metalloproteinase (MMP) activity is associated with matrix remodeling and cell migration and so increased MMP activity would suggest presence of an activated proscarring cell type.

Zymography. Gelatin zymography was used to assess MMP-9 and -2 activities. Conditioned medium $(5 \mu \mathrm{L})$ was mixed 1:1 with sample buffer and loaded onto a zymogram gel. Electrophoresis was performed at $125 \mathrm{~V}$ for $90 \mathrm{~min}$. The gel was incubated with zymogram renaturing buffer and the developing buffer as per the manufacturer's instructions. The next day, the gel was stained with $0.01 \%$ Coomassie Blue (Bio-Rad) in $40 \%$ ethanol/10\% acetic acid for $30 \mathrm{~min}$ before destaining, using $10 \%$ ethanol/7.5\% acetic acid for $1 \mathrm{~h}$. A photo was taken for qualitative evaluation.

Only 13 samples could be run on each gel and so for qualitative comparison between hLE - and hLE + RAFT TEs, and nonairlifted and airlifted RAFT TEs, samples from each single experiment (either normal hLF or dFib) were compared on one gel. To compare normal hLF and dFib directly, conditioned medium from each separate experiment was pooled and compared on one gel (either from airlifted or nonairlifted RAFT TEs).

Enzyme-linked immunosorbent assay. An enzymelinked immunosorbent assay (ELISA) kit (DMP100; R\&D Biosystems) was used to quantify MMP-1 secretion from conditioned medium according to the manufacturer's in- structions. Optical density $(450 \mathrm{~nm})$ was measured using a plate reader (MTX Lab Systems, Inc.) and data analyzed using the Excel Software. Appropriate blanks were included in each experiment.

\section{Transforming growth factor- $\beta 1$ secretion}

Transforming growth factor- $\beta 1$ (TGF- $\beta 1$ ) is a soluble factor that can trigger transdifferentiation of quiescent fibroblasts into an activated pro-scarring cell type and so increased levels of TGF- $\beta 1$ would indicate an increased likelihood of pro-scarring cells.

An ELISA kit (KAC1688) was used to quantify TGF- $\beta 1$ in conditioned medium according to the manufacturer's instructions. Conditioned medium was concentrated five times before assay using centrifugal filters (Millipore). Optical density $(450 \mathrm{~nm})$ was measured and analyzed as above.

\section{Collagen production}

De novo collagen production is associated with stromal and basement membrane remodeling, characteristic of proscarring cells; increased collagen synthesis would, therefore, indicate a greater number of activated, pro-scarring cells.

Collagen production in conditioned medium samples was quantified using a Sircol assay kit (Biocolor). All conditioned media samples were concentrated using the optional isolation and collagen concentration step included within the kit. Optical density $(555 \mathrm{~nm})$ was measured and analyzed as above.

\section{Wounding hLE on RAFT TE}

To determine whether or not wounding the epithelium affected normal hLF phenotype, hLE on RAFT TEs were wounded and normal hLF characterized using immunohistochemistry.

Nonairlifted normal hLF RAFT TEs with hLE were wounded by the application of heptanol-soaked paper discs for $1 \mathrm{~min}$. After wounding, RAFT TEs were returned to the airlifting culture to mimic in vivo conditions (i.e., the front of the eye). RAFT TEs were fixed for immunohistochemistry either immediately before or 6 days after wounding.

\section{Wholemount immunohistochemistry against $\alpha$-smooth muscle actin}

$\alpha$-Smooth muscle actin ( $\alpha$-SMA) expression suggests the presence of myofibroblasts and that a contractile scarring response is underway.

Creation of immunohistochemistry positive control $\alpha$-SMAexpressing cells. Normal hLF or dFib were seeded into $200 \mu \mathrm{L}$ tethered collagen gels (i.e., produced as RAFT TEs, but without application of absorbers) and cultured for $48 \mathrm{~h}$ to force transdifferentiation into myofibroblasts under mechanical tension, ${ }^{14,15}$ before fixing for $1 \mathrm{~h}$ in $4 \%$ paraformaldehyde (PFA; VWR) in PBS.

Wholemount immunohistochemistry staining. RAFT TEs were fixed in $4 \%$ PFA for $30 \mathrm{~min}$. Samples were washed with PBS and blocked for $1 \mathrm{~h}$ in $5 \%$ normal goat serum with $0.25 \%$ Triton X. Samples were washed with PBS before 
simultaneous application of a Cy3-conjugated $\alpha$-SMA antibody (1:200; Sigma-Aldrich) and FITC-phalloidin (1:1000; Sigma-Aldrich) in blocking buffer for $1 \mathrm{~h}$ in the dark. Negative control samples were obtained by the omission of Cy3-conjugated antibody. Samples were washed with PBS and transferred onto microscope slides. Nuclei were counterstained using DAPI mounting medium (Vector Labs). Coverslips were applied before analysis on a LSM 710 microscope (Zeiss).

Three fields of view on each sample were captured at $40 \times$ magnification for qualitative evaluation of $\alpha$-SMA expression. Using the micrographs, cells were identified as either positive or negative for $\alpha$-SMA and scored as follows: $0-1$ positive cells out of 10 were scored as,$- 2-4$ positive cells out of 10 as,$+ 5-7$ positive cells out of 10 as ++ , and 8 or more positive cells out of 10 as +++ .

\section{Statistics}

Within each condition within each experiment, three RAFT TEs were tested. Each experiment was performed in triplicate using cells isolated from six different fibroblast donors (i.e., three normal hLF and three dFib) and three different hLE donors. Data are presented as averages of three individual experiments \pm standard deviation. GraphPad Prism was used for statistical analyses. Where two groups were compared, unpaired $t$-tests were used. Where more than two groups were compared, a one-way analysis of variance (ANOVA) was used. Tukey's multiple comparisons test was used to test for individual comparisons following ANOVA. $p<0.05$ was considered statistically significant.

\section{Results}

MMP-9 and MMP-2 activity is increased in $d F i b$ compared with normal $h L F$, and is affected by the presence of $h L E$ and by airlifting

MMP activity in both normal hLF and dFib RAFT TEs was affected by the presence of hLE and by airlifting (Fig. 1A). Pro MMP-9 (92 kDa) was only found when hLE were present on RAFT TE. MMP-9 activity was lower in airlifted RAFT TEs compared with nonairlifted RAFT TEs. Both pro MMP-2 (66 kDa) and active MMP-2 (62 kDa) activity was found in all RAFT TEs. The presence of hLE reduced MMP-2 activity in both normal and dFib RAFT TEs. Conversely, airlifting increased MMP-2 activity in both normal and dFib RAFT TEs.

When MMP activity in normal hLF and positive control dFib RAFT TEs was compared directly (Fig. 1B) on the same zymogram before airlifting, activities of pro MMP-9 and both forms of MMP-2 was increased in dFib RAFT TEs compared with normal hLF RAFT TEs. However, after airlifting, there were no differences in MMP activities between normal hLF and dFib RAFT TEs.

Pro MMP-1 secretion did not differ between normal $h L F$ and dFib RAFT TEs and was unaffected by the presence of hLE or by airlifting

There was no significant difference in the production of pro MMP-1 in positive control dFib RAFT TEs compared with normal hLF RAFT TEs in the presence or absence of hLE or airlifting (Fig. 2).
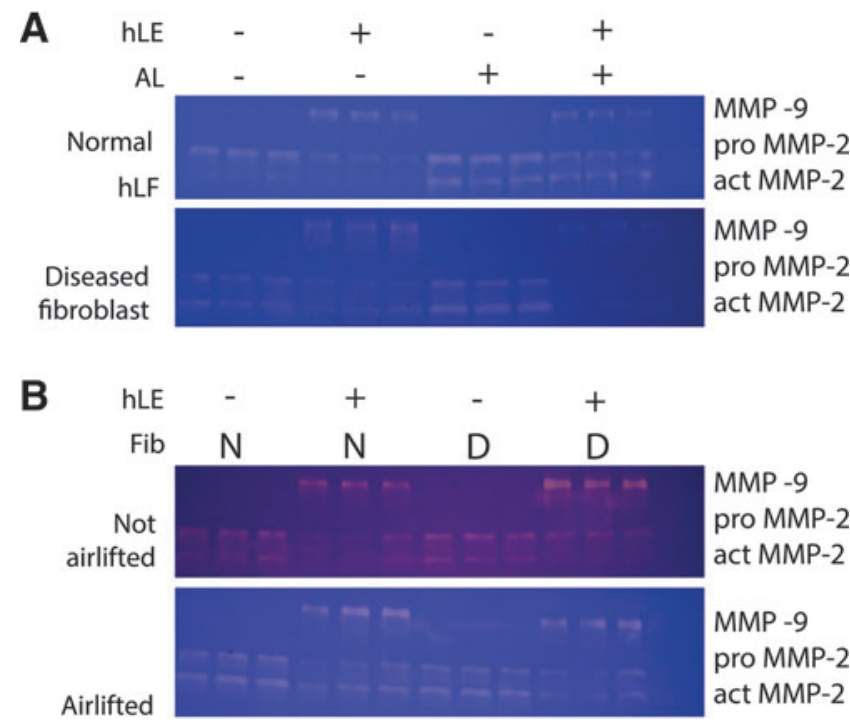

FIG. 1. MMP-2 and MMP-9 activity. MMP-2 and MMP-9 activity was measured from conditioned medium by gelatin zymography. The three bands on each zymogram correspond to pro MMP-9 (92 kDa), pro MMP-2 (66 kDa), and active MMP-2 (62 kDa) as labeled on the right of each gel. (A) MMP activity from RAFT TEs made with either normal hLF (top) or dFib (bottom) with or without hLE and from nonairlifted and airlifted RAFT TEs (AL). Each lane represents conditioned medium collected from a single RAFT TE construct within one experiment. The zymograms shown in (A) are representative of two further experimental repeats. (B) Comparison between MMP activities from RAFT TEs made with either normal hLF (N) or dFib (D) with hLE or without hLE. MMP activity was compared from nonairlifted (top) or airlifted (bottom) RAFT TEs. Each lane represents conditioned medium pooled from three separate experiments for each RAFT TE condition. In both $(\mathbf{A}, \mathbf{B})$, the right hand side lane represents the negative control (conditioned medium was collected from acellular RAFT TEs). dFib, diseased fibroblasts; hLE, human limbal epithelial cells; hLF, human limbal fibroblasts; MMP, matrix metalloproteinase; RAFT, real architecture for 3D tissue; TEs, tissue equivalents. Color images available online at www.liebertpub.com/tec

\section{Collagen production was increased from $d F i b$ \\ RAFT TES compared with normal hLF RAFT TES}

Collagen production from positive control dFib RAFT TEs was approximately three-fold greater than from normal hLF RAFT TEs $(p<0.05$, except for nonairlifted RAFT TEs without hLE) (Fig. 3). For all dFib RAFT TEs, over $7 \mu \mathrm{g}$ of collagen was measured in conditioned medium, compared with below $4 \mu \mathrm{g}$ of collagen for normal hLF RAFT TEs. For RAFT TEs populated with normal hLF, collagen production was unaffected by the presence of hLE or by airlifting (Fig. 3 ). Collagen production was significantly reduced from airlifted, dFib RAFT TEs with hLE compared with nonairlifted dFib RAFT TEs with hLE $(p<0.05)$ from $\sim 14$ to $\sim 7 \mu \mathrm{g}$.

\section{$\alpha-S M A$ expression was increased in dFib RAFT TES compared with normal hLF RAFT TEs}

The immunohistochemistry positive control $\alpha$-SMAexpressing cells in RAFT TEs (forced transdifferentiation 


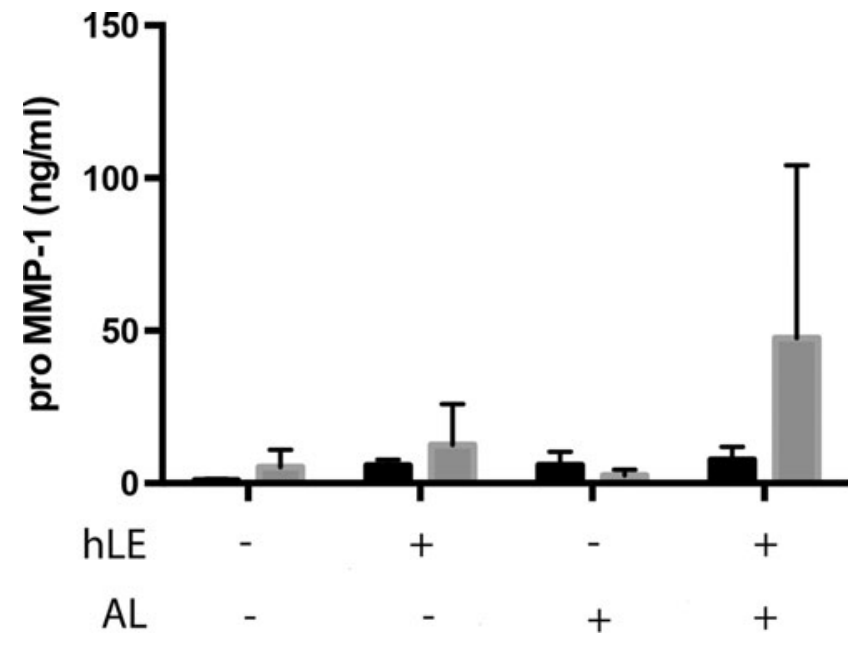

FIG. 2. Pro MMP-1 secretion. Pro MMP-1 secretion was measured from conditioned medium by ELISA. RAFT TE constructs contained either normal hLF (black bars) or dFib (gray bars). Conditioned medium was collected from RAFT TEs with or without hLE from nonairlifted and airlifted (AL) RAFT TEs. None of these results are statistically significant. $n=3 \pm \mathrm{SD}$. ELISA, enzyme-linked immunosorbent assay; SD, standard deviation.

under mechanical tension) (Fig. 4A) exhibited a dendritic morphology with strong $\alpha$-SMA expression. In the negative control RAFT TEs (antibody omitted) (Fig. 4B), no positive $\alpha$-SMA staining was observed.

In RAFT TEs populated with normal hLF (Fig. 4C-F), there was very little $\alpha$-SMA expression. However, greater numbers of normal hLF were observed in hLE - RAFT TEs (Fig. 4C, E) and these hLF appeared disorganized with irregular cell morphologies. Normal hLF in hLE + RAFT TEs (Fig. 4D, F) appeared sparser and were more regular in morphology.

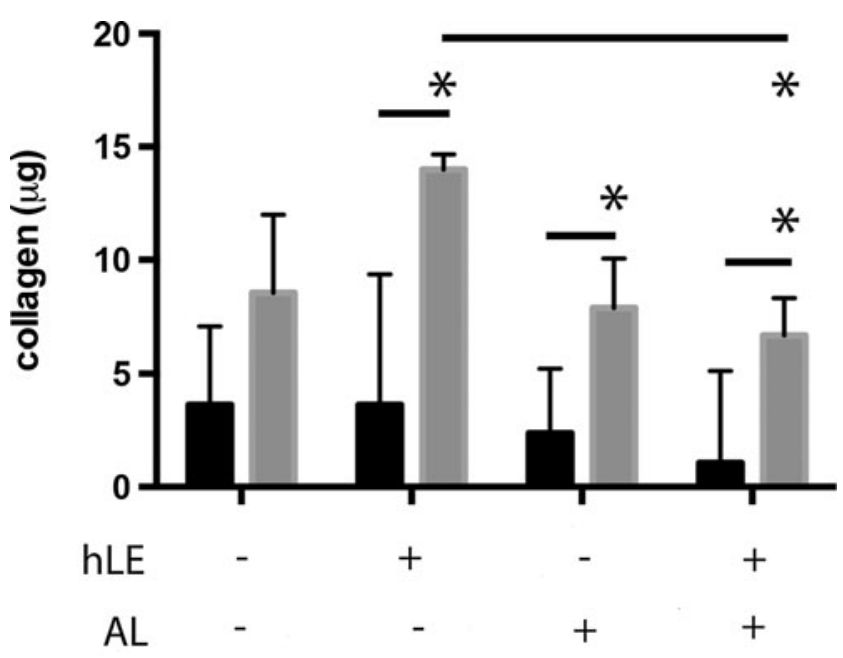

FIG. 3. Collagen production. Collagen production was measured from conditioned medium by the Sircol colorimetric assay. RAFT TE constructs contained either normal hLF (black bars) or dFib (gray bars). Conditioned medium was collected from RAFT TEs with or without hLE from nonairlifted and airlifted (AL) RAFT TEs. ${ }^{*} p<0.05 . n=3 \pm$ SD.
In positive control dFib RAFT TEs (Fig. 4G-J) many more cells expressed $\alpha$-SMA compared with normal hLF RAFT TEs. Again, greater numbers of $\mathrm{dFib}$ were present in the hLE - RAFT TEs (Fig. 4G, I) compared with hLE + RAFT TEs (Fig. 4H, J). Although nearly all of these dFib expressed $\alpha$-SMA, they did not exhibit the typical square myofibroblast morphology, and there was little evidence of stress fiber assembly. In hLE - RAFT TEs (Fig. 4H, J), a smaller proportion of $\mathrm{dFib}$ expressed $\alpha$-SMA, but those that did displayed a more typical square myofibroblast morphology, with evidence of stress fiber assembly. These results are summarized in Table 2.

\section{TGF- $\beta 1$ secretion was similar from both normal hLF RAFT TEs and dFib RAFT TES}

TGF- $\beta 1$ secretion was similar from normal hLF and positive control dFib populated RAFT TEs (Fig. 5). However, for both types of fibroblast, there was a significant trend toward decreased TGF- $\beta 1$ secretion from airlifted RAFT TEs compared with nonairlifted RAFT TEs, regardless of the presence or absence of hLE $(p<0.05)$. Before airlifting, TGF- $\beta 1$ was quantified at above $100 \mathrm{pg} / \mathrm{mL}$, reduced to below $\sim 50 \mathrm{pg} / \mathrm{mL}$ after airlifting for both normal hLF and dFib.

\section{Epithelial injury did not cause activation of normal $h L F$ in RAFT TEs to express $\alpha-S M A$}

Before hLE wounding, there was very little $\alpha$-SMA expression by normal hLF in RAFT TE (Fig. 6A) and no evidence of stress fiber assembly, in agreement with the results described in Figure 4. Six days postwounding, there were similarly low levels of $\alpha$-SMA expression, and cell morphology remained unaltered (Fig. 6B).

\section{Discussion}

LESC deficiency can cause ocular surface failure and blindness. We have developed RAFT TEs that could be used as a carrier to transplant hLE. hLE organization and basement membrane production is enhanced on RAFT TEs when hLF are present. ${ }^{9}$ However, hLF have the potential to differentiate into myofibroblast-like cells. The aim of this study was to determine if normal hLF in RAFT TEs are proscarring or not, to determine whether hLF may be safe to transplant alongside hLE.

We chose MMP activities, de novo collagen synthesis, $\alpha-$ SMA expression, and TGF- $\beta$ secretion as surrogate scarring parameters. Significant upregulation of any/all of these would suggest that the fibroblasts might be becoming myofibroblast-like ${ }^{16-18}$ and similar to those observed as part of a normal wound healing response. Normally, when no longer needed, myofibroblasts should apoptose to restore quiescence to the stroma. ${ }^{19}$ However, this is incorrectly regulated in Oc-MMP patients and the resultant persistent scarring response can cause blindness. dFib from Oc-MMP patients are known to retain pro-scarring parameters in $2 \mathrm{D}$ cultures (proliferation, migration, collagen contraction and synthesis, MMP, and eotaxin expression are all increased in dFib compared with normal conjunctival fibroblasts) ${ }^{12}$ so we exploited $\mathrm{dFib}$ as a positive control comparator for normal hLF. As stated earlier, unfortunately pro-scarring limbal fibroblasts are unavailable as biopsying them would be detrimental to the patient. 
Controls
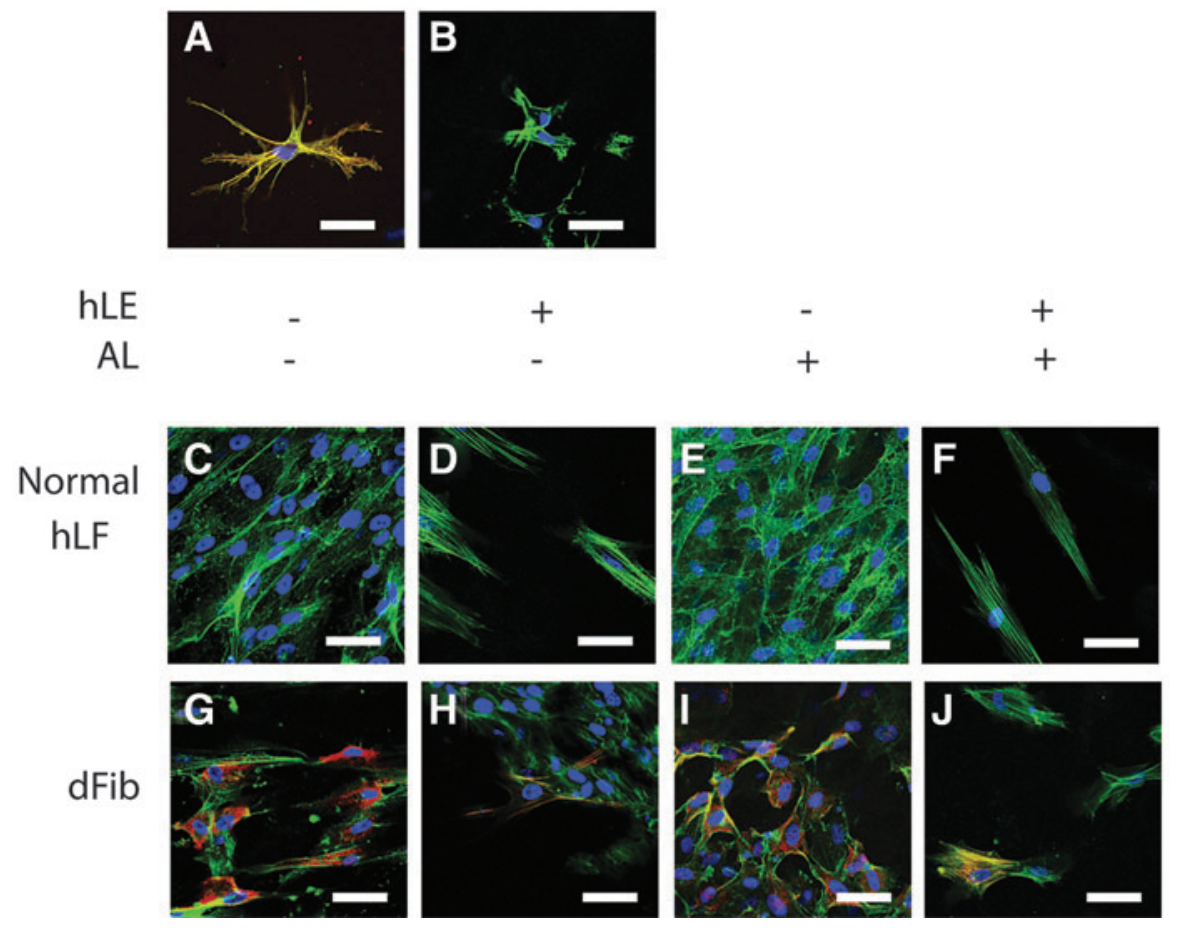

FIG. 4. $\alpha$-SMA expression. Fibroblast expression of $\alpha$-SMA (red) in RAFT TE constructs was observed using wholemount immunohistochemistry. RAFT TE constructs were also stained for phalloidin (green) and nuclei were counterstained using DAPI (blue). The positive (forced differentiation under mechanical tension) and negative (omission of anti- $\alpha$-SMA antibody) controls are shown in (A, B), respectively (top). (C-F) Staining of normal hLF within RAFT TE (middle). (G-J) Staining of dFib within RAFT TE (bottom). (D, F, H, J) hLE + RAFT TEs (C, E, G, I: hLE - RAFT TEs). (E, F, I, J) Airlifted RAFT TEs (C, D, G, H: nonairlifted RAFT TEs). All images are representative of three fields of view captured per condition per experiment. Scale bars: $50 \mu \mathrm{m} . \alpha$-SMA, $\alpha$-smooth muscle actin. Color images available online at www.liebertpub.com/tec

MMP activity is associated with matrix remodeling and cell migration. (MMP-1 is required to cleave the collagen triple helix so that MMP-2 or -9 can access their respective gelatin substrate cleavage sites). We found that MMP-9 and -2 activities were greater for $\mathrm{dFib}$ than for normal hLF before airlifting, which is the first indicator that normal hLF remain less activated than dFib in RAFT TEs, which is encouraging.

More specifically, MMP-9 is primarily associated with basement membrane remodeling by and migration of, hLE, (reviewed in Sivak and Fini ${ }^{20}$ ), and in agreement, we only saw MMP-9 when hLE were present. MMP-9 activity suggests that the hLE are remodeling basement membrane/ migrating, which is to be expected given that these cells are establishing a new culture on RAFT TEs. Interestingly, MMP-9 activity was decreased following airlifting. This suggests that the requirement for further remodeling (and

TABle 2. Summary of $\alpha$-SMA ExPRESSION IN NORMAL HLF AND DFIB RAFT TES

\begin{tabular}{lcccc}
\hline & $\begin{array}{c}\text { Non-AL, } \\
h L E-\end{array}$ & $\begin{array}{c}\text { Non-AL, } \\
h L E+\end{array}$ & $\begin{array}{c}A L, \\
h L E-\end{array}$ & $\begin{array}{c}A L, \\
h L E+\end{array}$ \\
\hline $\begin{array}{l}\text { Normal hLF } \\
\text { dFib }\end{array}$ & - & - & - & - \\
\hline
\end{tabular}

- indicates $0-1 \alpha$-SMA positive cells out of 10 .

+ indicates 2-4 $\alpha$-SMA positive cells out of 10 .

+++ indicates 8 or more $\alpha$-SMA positive cells out of 10 .

This scoring system is described in the Materials and Methods section.

AL, airlifted; $\alpha$-SMA, $\alpha$-smooth muscle actin; dFib, human conjunctival fibroblasts isolated from donors with ocular mucous membrane pemphigoid; hLE, human limbal epithelial cells; hLF, human limbal fibroblasts; RAFT, real architecture for 3D tissue; TEs, tissue equivalents.
MMP-9) was reduced at this later stage of culture when, possibly, hLE are more established.

Conversely, MMP-2 is primarily concerned with stromal remodeling by fibroblasts (reviewed in Sivak and Fini ${ }^{20}$ ). The upregulation of MMP-2 by both normal hLF and dFib following airlifting suggests that both are continuing to remodel the RAFT TE at this stage (perhaps so as to achieve a closer resemblance to native stroma). Interestingly, MMP-2 activity

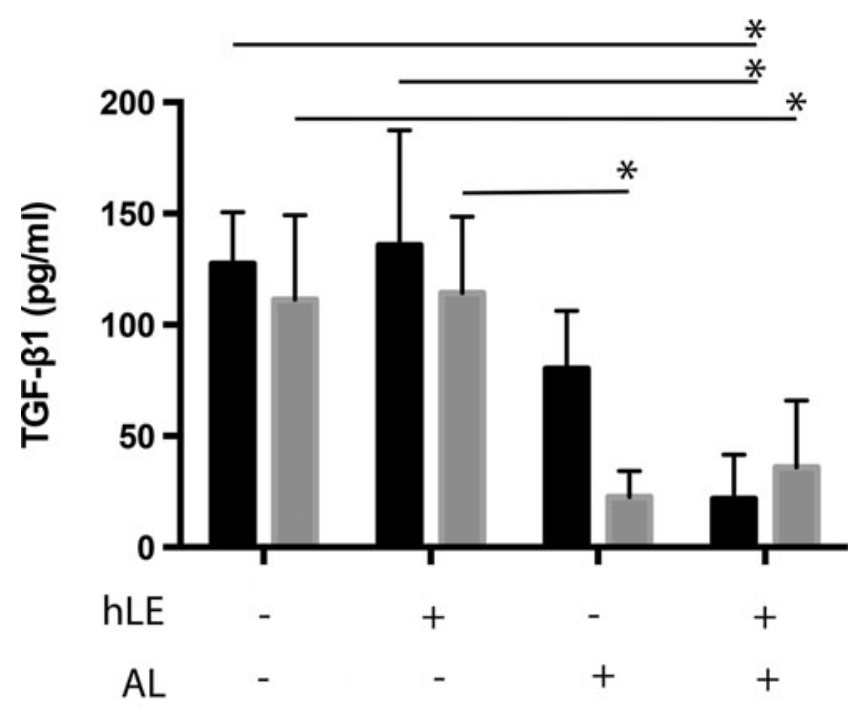

FIG. 5. TGF- $\beta 1$ secretion. TGF- $\beta 1$ secretion was measured from conditioned medium by ELISA. RAFT TE constructs contained either normal hLF (black bars) or dFib (gray bars). Conditioned medium was collected from RAFT TEs with or without hLE from nonairlifted and airlifted (AL) RAFT TEs. $* p<0.05 . n=3 \pm$ SD. TGF- $\beta 1$, transforming growth factor- $\beta 1$. 

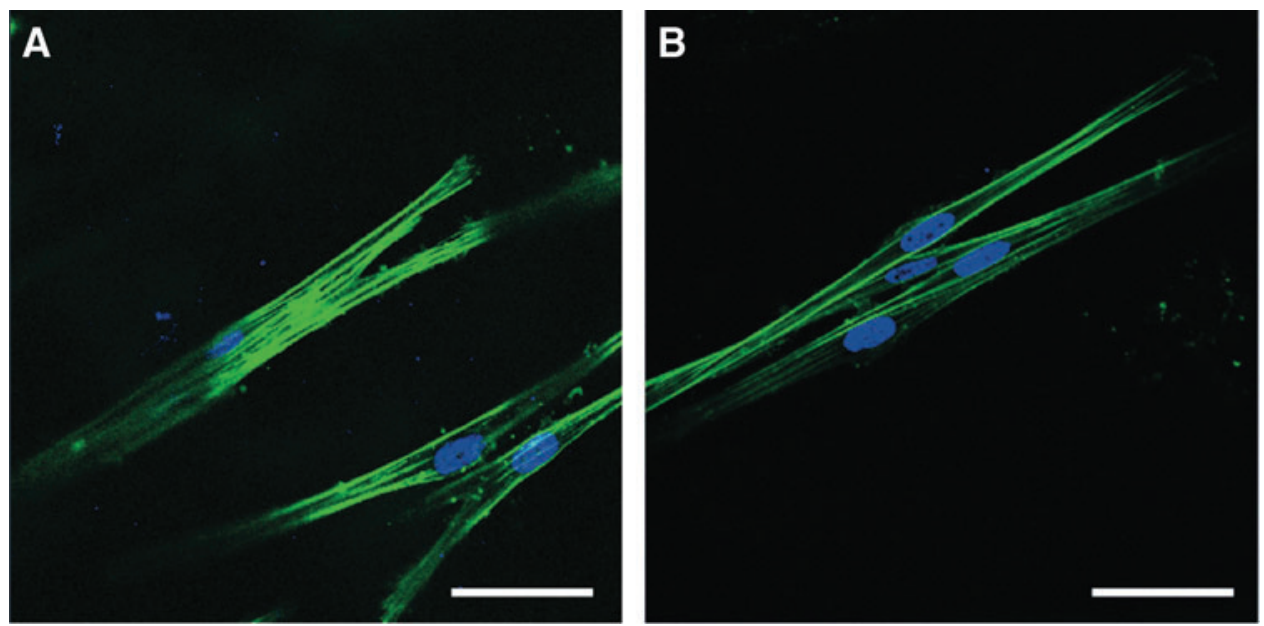

FIG. 6. Normal hLF response in RAFT TE to hLE wound. Expression of $\alpha$-SMA (red) in normal hLF in nonairlifted, hLE + RAFT TE constructs was observed by wholemount immunohistochemistry. RAFT TE constructs were also stained for phalloidin (green) and nuclei were counterstained using DAPI (blue). RAFT TE constructs were stained either immediately before $(\mathbf{A})$, or 6 days after $(\mathbf{B})$, wounding. Images are representative of five fields of view captured per condition per experiment. Scale bars: $50 \mu \mathrm{m}$. Color images available online at www.liebertpub.com/tec

was suppressed by the presence of hLE for both fibroblast types, suggesting that the presence of hLE quietens the activated phenotype. Potentially, without hLE, the lack of crosstalk deceives the fibroblasts into experiencing an artificial wounding environment, thus promoting the activated phenotype. To treat LESC deficiency, we would want to transplant RAFT TEs with hLE and so this finding is encouraging.

Collagen production is also associated with stroma and basement membrane remodeling. ${ }^{21,22}$ Again, we observed greater collagen production from dFib RAFT TEs when compared with normal hLF RAFT TEs, further suggesting that normal hLF are less activated than dFib. For normal $\mathrm{hLF}$, the amounts of collagen produced were unaffected by the presence or absence of hLE or by airlifting. For dFib, we found that collagen production was decreased after airlifting, compared with before. This appears counterintuitive to MMP-2 activities (which were increased after airlifting), but this may reflect that the RAFT TE is simply being remodeled at this stage of culture, without a requirement for continual deposition of new collagen.

The presence of myofibroblasts suggests a contractile scarring response is underway. Myofibroblasts appear larger and squarer than fibroblasts, with contractile stress fibers and have altered gene expression patterns, notably upregulation of $\alpha$-SMA. ${ }^{23-26}$ Transdifferentiation to myofibroblasts is mediated by mechanical stress ${ }^{27,28}$ (as exploited to achieve our $\alpha$-SMA positive controls at this point) and/or cytokine release following injury. ${ }^{29,30}$

We found higher levels of $\alpha$-SMA expression in dFib than normal hLF, which in agreement with MMP activity and collagen production data, suggests that normal hLF are less activated than $\mathrm{dFib}$ in RAFT TEs. However, $\alpha$-SMA-positive $\mathrm{dFib}$ in hLE - RAFT TEs did not display the typical myofibroblast morphology, but instead appeared more protomyofibroblast-like (the intermediate cell type between fibroblasts and myofibroblasts). ${ }^{31,32}$ Potentially, the differentiation process was unable to complete within the 2-3 week culture period, and/or entrapment within RAFT TEs affects the transdifferentiation process.
Immunohistochemistry also revealed that for both dFib and normal hLF fibroblast cell density within RAFT TEs was decreased when hLE were present. Furthermore for dFib, we found that $\alpha$-SMA expression was suppressed by hLE. Decreased $\alpha$-SMA expression suggests reduced activation when hLE are present, compared with when they are absent (comparably with MMP-2 activity). Again, as we would want to transplant RAFT TE with hLE, that hLE additionally suppress $\alpha$-SMA expression is favorable.

That fibroblast cell density is affected by hLE is important with respect to MMP activity as the observed differences may result from differing fibroblast numbers. However, the differential expression of $\alpha$-SMA suggests that hLE do impact on fibroblast phenotype, presumably as a result of crosstalk (or lack of). Previous work demonstrating that hLF enhance hLE phenotype in RAFT TEs provides evidence of such crosstalk. ${ }^{9}$ Fibroblast/epithelial crosstalk has also been shown to occur in another similar 3D format (collagen hydrogels). ${ }^{33}$ This further corroborates the possibility that fibroblast phenotype may be affected by, and alter in response to, hLE in RAFT TE.

As a final surrogate scarring parameter, we looked at TGF- $\beta 1$ secretion. TGF- $\beta 1$ can trigger transdifferentiation of keratocytes into myofibroblasts in vivo, ${ }^{34-36}$ but also in 3D tissue-engineered constructs similar to RAFT TE, ${ }^{33,37}$ and so it was expected that higher levels of TGF- $\beta 1$ would be found in dFib RAFT TEs than normal hLF RAFT TEs. However, we did not find this to be the case and, in fact, the levels of TGF- $\beta 1$ from $\mathrm{dFib}$ and normal hLF RAFT TEs were almost identical.

There are a number of alternative soluble factors (not investigated in this study) that could account for the observed differences between normal hLF and dFib. Interleukins 4 and 5, and interferon- $\gamma$ have all been implicated in Oc-MMP patients, instead of TGF- $\beta .^{38}$ Additionally, in previous studies where TGF- $\beta$ expression was found to be increased in Oc-MMP tissue over normal tissue, tissue was biopsied and immediately fixed for immunohistochemistry, which is not comparable with this study. ${ }^{34-36}$ 
Interestingly, for both normal hLF and dFib RAFT TEs, TGF- $\beta 1$ secretion was reduced after airlifting. Significant manipulation of both fibroblast and epithelial cells (including culture in both $2 \mathrm{D}$ and in $3 \mathrm{D}$ ) is required to produce RAFT TEs, and this may promote a wounding phenotype, transiently increasing TGF- $\beta 1$ expression (i.e., before airlifting only). This may also, temporarily, mask any differences in TGF- $\beta$ expression between normal hLF and dFib.

The reduction in TGF- $\beta 1$ expression after airlifting could also provide mechanistic evidence for the decreased MMP-9 activity and collagen production after airlifting. Possibly, secreted factors from a more mature epithelium (i.e., airlifted) are less stimulatory than those from a growing epithelium (i.e., nonairlifted). This has been demonstrated previously: when fibroblasts were exposed to conditioned medium taken from a growing epithelium, migration and contraction was increased compared with fibroblasts exposed to conditioned medium from mature epithelium, ${ }^{39}$ again highlighting the importance of crosstalk between stromal and epithelial cells.

In summary, normal hLF remain relatively quiescent in RAFT even as dFib remain more activated. This maintenance of in vivo characteristics in vitro in RAFT TEs is important. First, in the context of treating LESC deficiency, these data suggest that RAFT TE containing normal hLF may be suitable for transplant alongside hLE, unlike dFib. Furthermore, normal hLF are quietest in airlifted, hLE + RAFT TEs. Obviously, when attempting to treat LESC deficiency, we would want to transplant RAFT TEs with hLE and our data suggests that normal hLF may be safe(st) to use when hLE are present. Additionally, we found no upregulation of $\alpha$-SMA expression by normal hLF in RAFT TE following hLE wounding. This was an unexpected result, as epithelial injury is known to trigger myofibroblast formation in vivo. ${ }^{30}$ It would, therefore, seem that, in vitro at least at this stage, there is no evidence to suggest that hLF are pro-scarring, which is encouraging. However, there are a number of possible caveats to this: first, the time scale of the experiment could have been too short and/or there was insufficient time for TGF- $\beta$ to accumulate (due to regular media changes) or the lack of direct contact between epithelial and stromal cells in RAFT TEs limits potential for transdifferentiation. In vivo studies will be required to determine whether or not normal hLF remain quiescent posttransplantation where (higher concentrations of) other soluble factors and cytokines are present, which may affect hLF differently. Nevertheless, these in vitro findings are encouraging.

Although not the main purpose of this study, that dFib maintain an activated phenotype in RAFT TEs, is also of interest as this suggests the potential use of RAFT TE as a $3 \mathrm{D}$ ex vivo disease model of Oc-MMP that could be used to test anti-scarring therapies. This is useful as an in vitro disease model of Oc-MMP is not currently in existence.

\section{Conclusions}

We assessed the scarring phenotype of normal hLF within RAFT TEs using MMP activity, de novo collagen synthesis, $\alpha$-SMA expression, and TGF- $\beta$ secretion as surrogate scarring parameters. We used dFib isolated from Oc-MMP patients as a positive control. We found that the two fibroblast types displayed very different phenotypes in RAFT TE: normal hLF remained quiescent by comparison with $\mathrm{dFib}$ meaning that there is not currently any reason to suggest that these cells may be unsafe to use clinically, although in vivo work is required to confirm this. As dFib maintained their activated, pro-scarring phenotype in 3D RAFT TEs, these could be utilized as an ex vivo disease model of Oc-MMP.

\section{Acknowledgments}

The authors would like to thank the Moorfields Eye Bank (United Kingdom) for assistance with procurement of donor corneal tissue. The authors would also like to thank Prof. John K. Dart for obtaining biopsies from Oc-MMP patients and for reading the article. This study was supported by the Technology Strategy Board (I.M., J.T.D.), Fight for Sight (S.D.B., J.T.D.), and the National Institute for Health Research (NIHR) Biomedical Research Centre for Ophthalmology, Moorfields Eye Hospital and UCL Institute of Ophthalmology (J.T.D.).

\section{Disclosure Statement}

I.M. and S.B.D. declare that no competing financial interests exist; J.T.D. holds peer-reviewed funding for the development of the RAFT process.

\section{References}

1. Forrester, J.V. The Eye: Basic Sciences in Practice, 2nd edition. New York: W.B. Saunders, 2001.

2. Shortt, A.J., Secker, G.A., Munro, P.M., Khaw, P.T., Tuft, S.J., and Daniels, J.T. Characterization of the limbal epithelial stem cell niche: novel imaging techniques permit in vivo observation and targeted biopsy of limbal epithelial stem cells. Stem Cells 25, 1402, 2007.

3. Thoft, R., and Friend, J. The X, Y, Z hypothesis of corneal epithelial maintenance. Invest Ophthalmol Vis Sci 10, 1442, 1983.

4. Dua, H.S., Saini, J.S., Azuara-Blanco, A., and Gupta, P. Limbal stem cell deficiency: concept, aetiology, clinical presentation, diagnosis and management. Indian J Ophthalmol 48, 83, 2000.

5. Kolli, S., Ahmad, S., Lako, M., and Figueiredo, F. Successful clinical implementation of corneal epithelial stem cell therapy for treatment of unilateral limbal stem cell deficiency. Stem Cells 28, 597, 2010.

6. Nakamura, T., Sotozono, C., Bentley, A.J., Mano, S., Inatomi, T., Koizumi, N., Fullwood, N.J., and Kinoshita, S. Long-term phenotypic study after allogeneic cultivated corneal limbal epithelial transplantation for severe ocular surface diseases. Ophthalmology 117, 2247, 2010.

7. Prabhasawat, P., Ekpo, P., Uiprasertkul, M., Chotikavanich, S., and Tesavibul, N. Efficacy of cultivated corneal epithelial stem cells for ocular surface reconstruction. Clin Ophthalmol 6, 1483, 2012.

8. Connon, C.J., Doutch, J., Chen, B., Hopkinson, A., Mehta, J.S., Nakamura, T., Kinoshita, S., and Meek, K.M. The variation in transparency of amniotic membrane used in ocular surface regeneration. Br J Ophthalmol 94, 1057, 2010.

9. Levis, H.J., Brown, R.A., and Daniels, J.T. Plastic compressed collagen as a biomimetic substrate for human limbal epithelial cell culture. Biomaterials 31, 7726, 2010.

10. Zieske, J.D., Guimaraes, S.R., and Hutcheon, A.E. Kinetics of keratocyte proliferation in response to epithelial debridement. Exp Eye Res 72, 33, 2001. 
11. Chan, L.S. Ocular and oral mucous membrane pemphigoid (cicatricial pemphigoid). Clin Dermatol 30, 34, 2012.

12. Saw, V.P., Schmidt, E., Offiah, I., Galatowicz, G., Zillikens, D., Dart, J.K., Calder, V.L., and Daniels, J.T. Profibrotic phenotype of conjunctival fibroblasts from mucous membrane pemphigoid. Am J Pathol 178, 187, 2011.

13. Levis, H.J., Massie, I., Dziasko, M.A., Kaasi, A., and Daniels, J.T. Rapid tissue engineering of biomimetic human corneal limbal crypts with 3D niche architecture. Biomaterials 34, 8860, 2013.

14. Arora, P.D., Narani, N., and McCulloch, C.A. The compliance of collagen gels regulates transforming growth factor-beta induction of alpha-smooth muscle actin in fibroblasts. Am J Pathol 154, 871, 1999.

15. Garrett, Q., Khaw, P.T., Blalock, T.D., Schultz, G.S., Grotendorst, G.R., and Daniels, J.T. Involvement of CTGF in TGF-beta1-stimulation of myofibroblast differentiation and collagen matrix contraction in the presence of mechanical stress. Invest Ophthalmol Vis Sci 45, 1109, 2004.

16. El-Shabrawi, Y., Kublin, C.L., and Cintron, C. mRNA levels of alpha1(VI) collagen, alpha1(XII) collagen, and beta ig in rabbit cornea during normal development and healing. Invest Ophthalmol Vis Sci 39, 36, 1998.

17. Matsubara, M., Girard, M.T., Kublin, C.L., Cintron, C., and Fini, M.E. Differential roles for two gelatinolytic enzymes of the matrix metalloproteinase family in the remodelling cornea. Dev Biol 147, 425, 1991.

18. Tandon, A., Tovey, J.C., Sharma, A., Gupta, R., and Mohan, R.R. Role of transforming growth factor Beta in corneal function, biology and pathology. Curr Mol Med 10, 565, 2010.

19. Wilson, S.E., Chaurasia, S.S., and Medeiros, F.W. Apoptosis in the initiation, modulation and termination of the corneal wound healing response. Exp Eye Res 85, 305, 2007.

20. Sivak, J.M., and Fini, M.E. MMPs in the eye: emerging roles for matrix metalloproteinases in ocular physiology. Prog Retin Eye Res 21, 1, 2002.

21. Davison, P.F., and Galbavy, E.J. Connective tissue remodeling in corneal and scleral wounds. Invest Ophthalmol Vis Sci 27, 1478, 1986.

22. Ishizaki, M., Shimoda, M., Wakamatsu, K., Ogro, T., Yamanaka, N., Kao, C.W., and Kao, W.W. Stromal fibroblasts are associated with collagen IV in scar tissues of alkaliburned and lacerated corneas. Curr Eye Res 16, 339, 1997.

23. Jester, J.V., Barry-Lane, P.A., Cavanagh, H.D., and Petroll, W.M. Induction of alpha-smooth muscle actin expression and myofibroblast transformation in cultured corneal keratocytes. Cornea 15, 505, 1996.

24. Jester, J.V., Petroll, W.M., Barry, P.A., and Cavanagh, H.D. Expression of alpha-smooth muscle (alpha-SM) actin during corneal stromal wound healing. Invest Ophthalmol Vis Sci 36, 809, 1995.

25. Jester, J.V., Petroll, W.M., and Cavanagh, H.D. Corneal stromal wound healing in refractive surgery: the role of myofibroblasts. Prog Retin Eye Res 18, 311, 1999.

26. West-Mays, J.A., and Dwivedi, D.J. The keratocyte: corneal stromal cell with variable repair phenotypes. Int $\mathrm{J}$ Biochem Cell Biol 38, 1625, 2006.
27. Junker, J.P., Kratz, C., Tollback, A., and Kratz, G. Mechanical tension stimulates the transdifferentiation of fibroblasts into myofibroblasts in human burn scars. Burns 34, 942, 2008.

28. Wang, J., Chen, H., Seth, A., and McCulloch, C.A. Mechanical force regulation of myofibroblast differentiation in cardiac fibroblasts. Am J Physiol Heart Circ Physiol 285, H1871, 2003.

29. Saika, S., Yamanaka, O., Sumioka, T., Miyamoto, T., Miyazaki, K., Okada, Y., Kitano, A., Shirai, K., Tanaka, S., and Ikeda, K. Fibrotic disorders in the eye: targets of gene therapy. Prog Retin Eye Res 27, 177, 2008.

30. Wilson, S.E. Corneal myofibroblast biology and pathobiology: generation, persistence, and transparency. Exp Eye Res 99, 78, 2012.

31. Desmouliere, A., Chaponnier, C., and Gabbiani, G. Tissue repair, contraction, and the myofibroblast. Wound Repair Regen 13, 7, 2005.

32. Tomasek, J.J., Gabbiani, G., Hinz, B., Chaponnier, C., and Brown, R.A. Myofibroblasts and mechano-regulation of connective tissue remodelling. Nat Rev Mol Cell Biol 3, 349, 2002.

33. Wilson, S.L., Yang, Y., and El Haj, A.J. Corneal stromal cell plasticity: in vitro regulation of cell phenotype through cell-cell interactions in a three-dimensional model. Tissue Eng Part A 20, 225, 2013.

34. Bernauer, W., Wright, P., Dart, J.K., Leonard, J.N., and Lightman, $\mathrm{S}$. The conjunctiva in acute and chronic mucous membrane pemphigoid. An immunohistochemical analysis. Ophthalmology 100, 339, 1993.

35. Elder, M.J., Dart, J.K., and Lightman, S. Conjunctival fibrosis in ocular cicatricial pemphigoid-the role of cytokines. Exp Eye Res 65, 165, 1997.

36. Razzaque, M.S., Foster, C.S., and Ahmed, A.R. Role of collagen-binding heat shock protein 47 and transforming growth factor-beta1 in conjunctival scarring in ocular cicatricial pemphigoid. Invest Ophthalmol Vis Sci 44, 1616, 2003.

37. Karamichos, D., Hutcheon, A.E., and Zieske, J.D. Transforming growth factor-beta3 regulates assembly of a non-fibrotic matrix in a 3D corneal model. J Regen Med Tissue Eng 5, e228, 2011.

38. Caproni, M., Calzolari, A., Salvatore, E., Giomi, B., Volpi, W., D’Agata, A., Santucci, M., and Fabbri, P. Cytokine profile and supposed contribution to scarring in cicatricial pemphigoid. J Oral Pathol Med 32, 34, 2003.

39. Daniels, J.T., and Khaw, P.T. Temporal stimulation of corneal fibroblast wound healing activity by differentiating epithelium in vitro. Invest Ophthalmol Vis Sci 41, 3754, 2000.

Address correspondence to: Isobel Massie, PhD Department of Ocular Biology and Therapeutics UCL Institute of Ophthalmology 11-43 Bath Street London EC1V 9EL United Kingdom

E-mail: i.massie@ucl.ac.uk

Received: August 12, 2014 Accepted: November 5, 2014 Online Publication Date: December 16, 2014

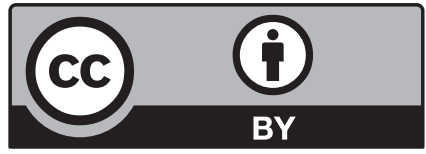

This work is licensed under a Creative Commons Attribution 3.0 United States License. You are free to copy, distribute, transmit and adapt this work, but you must attribute this work as "Tissue Engineering, Part C. Copyright 2015 Mary Ann Liebert, Inc. http://liebertpub.com/tec, used under a Creative Commons Attribution License: http:// creativecommons.org/licenses/by/3.0/us/” 